\title{
Acesso e utilização dos serviços na Estratégia Saúde da Família na perspectiva dos gestores, profissionais e usuários
}

\author{
Access to and use of the services of the family health strategy \\ from the perspective of managers, professionals and users
}

Regimarina Soares Reis ${ }^{1}$

Liberata Campos Coimbra ${ }^{1}$

Antônio Augusto Moura da Silva ${ }^{1}$

Alcione Miranda dos Santos ${ }^{1}$

Maria Teresa Seabra Soares de Britto e Alves ${ }^{1}$

Zeni Carvalho Lamy ${ }^{1}$

Sabrina Varão Oliveira Ribeiro ${ }^{1}$

Maria Socorro de Araújo Dias ${ }^{2}$

Raimundo Antonio da Silva ${ }^{1}$

${ }^{1}$ Centro de Ciências da Saúde, Universidade Federal do Maranhão. R. Viana Vaz s/n, Centro. 65.000-000 São Luís MA Brasil. regimarina.reis@gmail.com ${ }^{2}$ Universidade Estadual do Vale do Acaraú.
Abstract Questionnaires adapted to the Brazilian reality (Primary Care Assessment Tool) to evaluate access to and use of services were distributed to 30 managers, 80 professionals and a random sample of 882 Family Health Strategy (FHS) users in São Luís in the state of Maranhão. The differences between the evaluations of managers, professionals and users were identified by the Kruskal-Wallis test and Dunn's post test. Users faced difficulties in access to and use of the services, which were also considered unsatisfactory by managers and professionals. Access was the dimension with the worst evaluation, and non-functioning of units after 6 p.m. and at weekends were the main difficulties. The evaluations of the managers and professionals were almost always divergent from those of the users, with the evaluation of the managers being more favorable. The fact that the service was free of charge, the use of preventive services and the FHS services before the specialized care were well evaluated. The operation of units should attend users' needs, especially that of the working population. Strengthening social participation in local management of the FHS may assist in identifying these needs, thereby reducing the divergent opinions of the players involved.

Key words Family health program, Primary care, Health evaluation
Resumo Utilizaram-se questionários adaptados à realidade brasileira (PCATool) para avaliar acesso e utilização dos serviços, aplicados a 30 gestores, 80 profissionais e amostra aleatória de 882 usuários da Estratégia Saúde da Família (ESF) em São Luís. Diferenças entre as avaliações dos 3 tipos de entrevistados foram identificadas pelo teste de KruskallWallis e pós-teste de Dunn. Usuários tiveram dificuldades no acesso e na utilização dos serviços, também considerados insatisfatórios pelos gestores e profissionais. O acesso foi a dimensão pior avaliada e o não funcionamento das unidades após as 18 horas e finais de semana foram suas principais dificuldades. A avaliação dos gestores e profissionais quase sempre divergiu com a dos usuários, sendo a avaliação dos gestores predominantemente mais favorável. Gratuidade, utilização de serviços preventivos e da ESF antes das consultas especializadas foram bem avaliadas. O funcionamento das unidades precisa se adequar às necessidades dos usuários, principalmente dos trabalhadores. Fortalecer a participação social na gestão local da ESF pode ajudar a identificar essas necessidades, dirimindo divergências entre os atores estudados. Palavras-chave Programa Saúde da Família, Atenção Primária, Avaliação em Saúde 


\section{Introdução}

O Sistema Único de Saúde (SUS) passa por um momento de reorganização pautado em investimentos na Estratégia Saúde da Família (ESF), que deve ser sua porta de entrada. A ideia de uma "porta" faz sentido, já que o usuário não conhece suficientemente os detalhes técnicos da atenção à saúde para avaliar de maneira adequada qual o momento e que tipo de atenção é apropriada ao seu problema ${ }^{1,2}$. Essa característica da ESF é conhecida como primeiro contato, acesso de primeiro contato, porta de entrada ou contato preferencial ${ }^{1,2}$.

Estabelecer um ponto de primeiro contato com o sistema de saúde, articular cuidados preventivos e curativos nesse primeiro nível de atenção e integrar as redes de cuidado primário e especializado são elementos fundamentais para organizar o SUS de forma integrada e possibilitar atenção de melhor qualidade e menos onero$\mathrm{sa}^{3}$. Desenvolver mecanismos de avaliação da capacidade e desempenho da ESF como porta de entrada do SUS faz-se importante, tanto para a consolidação das ações implementadas, quanto para fomentar o processo de discussão críticoreflexiva sobre a melhor proposta para organizar a atenção primária.

$O$ enfoque na ESF para a reorganização do modelo assistencial no Brasil foi pautado a partir da centralidade obtida pela Atenção Primária à Saúde (APS) nos anos anteriores à criação do SUS. No Brasil APS tem sido considerada atenção básica e hoje esses conceitos são tomados como sinônimos ${ }^{4,5}$.

Estudos observacionais e experimentais, nacionais e internacionais, demonstram o efeito da APS e seus atributos sobre a obtenção de melhores indicadores populacionais na saúde das crianças, sobre a redução das iniquidades no acesso ao sistema de saúde, sobre a satisfação dos usuários e sobre o aumento da efetividade e da eficiência da atenção sanitária ${ }^{6}$.

A concepção brasileira de APS vai ao encontro da definição elaborada por Starfield ${ }^{2}$, que define a APS como uma orientação do sistema de saúde para assegurar: a entrada no sistema para todas as novas necessidades e problemas de saúde; atenção sobre a pessoa de maneira longitudinal; atenção para todas as enfermidades, excetuando-se aquelas muito incomuns ou raras; e integração da atenção oferecida em outro nível. De tal conceito captam-se os quatro atributos ou características essenciais da APS: porta de entrada, longitudinalidade, integralidade e coordenação.
Para que a ESF possa se concretizar como "porta de entrada" é necessário que seus serviços sejam acessíveis e utilizados a cada nova necessidade ou problema de saúde 2 .

A "acessibilidade possibilita que as pessoas cheguem aos serviços. Ou seja, este é um aspecto da estrutura de um sistema ou unidade de saúde necessário para se atingir a atenção ao primeiro contato. "Acesso" é a forma como a pessoa experimenta esta característica de seu serviço de saúde"”.

Emprega-se o termo acessibilidade como caráter ou qualidade do que é acessível ou ambos os termos como sinônimos para indicar o grau de facilidade com que as pessoas obtêm cuidados de saúde 7 . Neste estudo, os termos acesso e acessibilidade são empregados como sinônimos.

Por sua vez, a utilização dos serviços de saúde compreende todo contato direto ou indireto com estes e é resultante da interação entre o comportamento dos indivíduos, os serviços disponíveis e os profissionais de saúde. O comportamento dos indivíduos influencia mais o primeiro contato com o serviço de saúde, enquanto as características dos profissionais influenciam mais a continuidade do cuidado ${ }^{8}$.

Trabalhos que avaliam a ESF como porta de entrada demonstram a fragilidade dessa característica, principalmente no que concerne ao aspecto estrutural - acesso aos serviços ${ }^{9,10}$. Segundo Giovanella et al. ${ }^{3}$, "a barreira de acesso mais importante é a disponibilidade e a presença física de serviços e recursos humanos, que representa condição necessária à utilização".

A necessidade de maiores informações sobre os aspectos organizacionais do acesso e uso de serviços de atenção básica em saúde e a escassez de trabalhos que considerem simultaneamente a perspectiva de gestores, profissionais e usuários, foram determinantes para a escolha deste tema. Assim, se buscou avaliar o acesso e a utilização da ESF como porta de entrada do SUS, identificando os elementos estruturais e processuais que fortalecem ou dificultam a concretização desse papel da ESF no município de São Luís, confrontando a visão de gestores, profissionais e usuários para identificar convergências e divergências.

\section{Métodos}

Trata-se de pesquisa avaliativa de abordagem quantitativa realizada de janeiro de 2010 a março de 2011 em São Luís. Este município, no ano de 2010, contava com 89 Equipes de Saúde da Fa- 
mília, distribuídas em 39 Unidades de Saúde, conferindo cobertura a $30,3 \%$ da população ${ }^{11,12}$.

\section{População e amostragem}

A população do estudo foi composta por gestores, profissionais e usuários da ESF em São Luís. Para a seleção dos gestores e profissionais não houve sorteio. Foram selecionados como sujeitos da pesquisa todos os 54 gestores (os 39 diretores das Unidades de Saúde da Família, os 12 supervisores de área da ESF, e os três coordenadores de nível central da Secretaria Municipal de Saúde - SEMUS), 79 médicos e 79 enfermeiros das equipes em funcionamento no período da coleta de dados. Para os gestores foi adotado como critério de inclusão ter pelo menos seis meses de experiência na função de gestão da ESF e para os profissionais ter atuação na equipe da ESF há pelo menos seis meses.

Dos gestores selecionados, 32 foram entrevistados, havendo participação de $64,1 \%$. Dos profissionais, $80(50,6 \%)$ foram entrevistados, sendo 22 médicos e 58 enfermeiros.

Para a seleção dos usuários, calculou-se o tamanho amostral tendo como parâmetros alfa 0,05 e poder do teste 0,8 . Foram acrescidos $10 \%$ para cobrir possíveis perdas, totalizando um tamanho amostral de 917. No cálculo foi necessário controlar a correlação intraclasse, multiplicando-se o tamanho da amostra pelo efeito do desenho. Utilizou-se como referência para o cálculo do valor da correlação o trabalho de Almeida e Macinko ${ }^{9}, \rho=0,25$.

Os usuários foram selecionados por meio de amostragem por conglomerados em duas etapas. Na primeira etapa foram selecionadas, por amostragem aleatória simples, 20 unidades de Saúde da Família do município, o que correspondia à metade daquelas em funcionamento. As 44 equipes de Saúde da Família em funcionamento nas unidades selecionadas foram incluídas no estudo. Na segunda etapa foram selecionados os usuários, sendo determinadas 21 entrevistas por equipe, o que totalizou 924 esperadas. Os usuários foram selecionados dentro das unidades de saúde em dias e turnos diferentes. Não se buscou tornar a amostra heterogênea por gênero ou idade. Ao final da coleta de dados foram entrevistados 882 usuários, havendo perda de $4,5 \%$.

Os usuários presentes na unidade de saúde no momento da visita foram incluídos no estudo sob os seguintes critérios, para aqueles com 18 anos de idade ou mais: estar cadastrado na
ESF há pelo menos seis meses, ter sido atendido anteriormente pela equipe e estar aguardando ou já ter recebido atendimento no momento da visita. Foram ainda incluídas todas as gestantes e mulheres que já tinham filhos, independente da idade, desde que atendessem aos demais critérios supracitados. Para os usuários menores de idade ou incapacitados o respondente do questionário foi o acompanhante, que deveria já ter acompanhado o usuário na Unidade em outra oportunidade e ter pelo menos 18 anos (exceção às mães menores acompanhando crianças).

\section{Instrumentos de Pesquisa}

Foram utilizados os questionários do Primary Care Assessment Tool (PCATool), instrumento formulado para mensurar as dimensões da atenção primária ${ }^{2,9}$, cuja validação no Brasil foi realizada por grupo de pesquisa da Universidade Federal do Rio Grande do Sul13,14. A versão utilizada na presente investigação possui seis opções de resposta, tendo sido adaptada à realidade brasileira em estudos realizados em Petrópolis ${ }^{9}$ e em São Paulo ${ }^{10}$. Em Petrópolis foi testada metodologia de avaliação rápida para medir as características organizacionais e de desempenho dos serviços de atenção básica no SUS a partir de dados secundários e informações obtidas dos gestores, profissionais e usuários por meio dos questionários do PCAtool adaptados. No Estado de São Paulo foi avaliado o desempenho da atenção básica em municípios com mais de 100 mil habitantes a partir de dados coletados com profissionais e usuários da atenção básica por meio dos mesmos instrumentos utilizados no estudo de Petrópolis ${ }^{9,10}$. Nesta pesquisa quatro tipos de questionários foram aplicados, de acordo com o respondente: usuários, acompanhantes, gestores e profissionais.

Os indicadores investigados sobre o acesso foram facilidade na marcação de consulta, horário de funcionamento das unidades, disponibilidade de telefone para informações, tempo de espera, medicamentos e equipamentos, e necessidade de pagamento para obtenção de atendimento. E os relacionados à utilização dos serviços foram necessidade de consulta na equipe de Saúde da Família antes de consultar especialista e o uso dos serviços preventivos e curativos.

Para a operacionalização deste estudo considerou-se o componente organizacional do acesso, portanto, foram avaliados obstáculos tipicamente relacionados à organização dos processos de trabalho, conforme propõe Donabedian ${ }^{7}$. 
Foram utilizadas seis opções de respostas para cada pergunta e essas opções eram iguais para cada tipo de entrevistado, conforme o Quadro 1.

Os escores e os percentuais de obtenção descritos estão de acordo com a proposição dos questionários adaptados à realidade brasileira em Petrópolis ${ }^{9}$. O grau de obtenção em três categorias foi estabelecido a partir do agrupamento dos tipos de resposta, conforme sua similaridade, o qual definiu os pontos de corte dos escores e percentuais de obtenção.

Embora o PCAtool seja um instrumento originado em outros contextos ${ }^{2,15}$ em que a APS se insere em sistemas de saúde organizados de forma diferente do SUS, os aspectos avaliados por esse instrumento no presente artigo (acesso e utilização dos serviços) estão coerentes e compatíveis com as propostas da ESF brasileira. Não foi identificada a necessidade de inserção ou retirada de indicadores no estudo.

\section{Coleta de dados}

Não puderam ser incluídos na pesquisa $12,7 \%$ dos profissionais e $12,8 \%$ dos gestores por não atenderem ao critério de inclusão; $15,8 \%$ dos profissionais e 7,7\% dos gestores que se recusaram a participar; $14,5 \%$ dos profissionais e $15,4 \%$ dos gestores com os quais não foi conseguido contato via telefone ou por visita nas Unidades de Saúde; e $6,4 \%$ dos profissionais que estavam de licença ou férias. Totalizou-se 32 gestores e 80 profissionais de fato entrevistados.

Ao todo foram entrevistados 649 usuários e 233 acompanhantes de usuários menores de idade ou incapacitados, totalizando 882 entrevistas.

$\mathrm{Na}$ análise dos dados os escores das perguntas sobre acesso foram somados e a média dessa soma representou o Índice Composto de Acesso.
Este mesmo processo foi seguido para construir o Índice Composto de Utilização dos Serviços. A partir da média da soma desses dois Índices Compostos foi construído o Índice Total da Porta de Entrada, que representa a experiência total da ESF em ser porta de entrada do SUS para a população cadastrada.

Foi calculado o alfa de Cronbach para cada dimensão (acesso e utilização dos serviços) a fim de se estimar a confiabilidade dos dados. A partir do alfa de Cronbach observou-se que a consistência interna foi maior nas respostas dos usuários e acompanhantes, variando de 0,47 a 0,58 para os quatro tipos de entrevistados.

A análise de consistência interna é o cálculo da correlação que existe entre cada item do teste e o restante dos itens ou o total (escore total) dos itens. São considerados valores adequados para o alfa de Cronbach, a partir de $0,60^{16,17}$. Porém, quando o número de itens é pequeno este parâmetro deve ser relativizado, visto que neste caso o próprio item em análise afeta substancialmente o escore total a seu favor ${ }^{13}$. Desse modo, considera-se que os valores encontrados do alfa foram adequados ao número restrito de itens em cada dimensão avaliada (acesso e utilização dos serviços).

Para detectar a existência de diferenças nos escores dos indicadores, índices compostos e índice total das diversas fontes de informação foi utilizado o teste de Kruskal-Wallis. Como pósteste foi utilizado o Teste de Dunn. O ponto de corte para significância estatística foi definido em $5 \%(\mathrm{p}<0,05)$. Foram utilizados os softwares STATA 10.0, EPI-INFO 3.2, e GraphPrism 5.0 para processamento e análise dos dados.

$\mathrm{Na}$ análise estatística os dados dos usuários maiores de 18 anos, menores de idade e incapacitados foram analisados conjuntamente e todos foram denominados usuários, embora coleta-

Quadro 1. Classificação do percentual e grau de obtenção dos componentes de acesso e utilização dos serviços da Estratégia Saúde da Família. São Luís, 2011.

\begin{tabular}{|l|c|c|c|c|c|c|}
\hline \multirow{2}{*}{\multicolumn{1}{|c|}{ Parâmetros }} & \multicolumn{6}{|c|}{ Tipos de resposta } \\
\cline { 2 - 7 } & Nunca & $\begin{array}{c}\text { Quase } \\
\text { nunca }\end{array}$ & $\begin{array}{c}\text { Algumas } \\
\text { vezes }\end{array}$ & $\begin{array}{c}\text { Muitas } \\
\text { vezes }\end{array}$ & $\begin{array}{c}\text { Quase } \\
\text { sempre }\end{array}$ & Sempre \\
\hline Escore & 0 & 1 & 2 & 3 & 4 & 5 \\
\hline Percentual de obtenção & $0 \%$ & $1 \%-20 \%$ & $21 \%-40 \%$ & $41 \%-60 \%$ & $61 \%-80 \%$ & $81 \%-100 \%$ \\
\hline Grau de obtenção & \multicolumn{2}{|c|}{ Crítico } & Insatisfatório & Satisfatório \\
\hline
\end{tabular}


dos em questionários diferentes, possibilitando análise mais ampla da experiência de utilização.

A Pesquisa foi aprovada pelo Comitê de Ética em Pesquisa da Universidade Federal do Maranhão. As entrevistas foram iniciadas somente após a leitura, concordância e assinatura do Termo de Consentimento Livre e Esclarecido, respeitandose os princípios éticos e legais da Resolução n ${ }^{\circ}$ 196/96 ${ }^{18}$ e suas complementares. Os dados foram coletados por equipe treinada em todas as etapas da pesquisa. Foi realizado estudo piloto em uma Unidade de Saúde do município, o que possibilitou ajustes pertinentes na pesquisa.

\section{Limitações}

Os baixos percentuais de participação dos profissionais e gestores foram limitações deste estudo. Isto pode sugerir a necessidade de compreensão da avaliação em saúde em seu caráter formativo e subsidiário do planejamento em vez de punitivo e burocrático. Segundo o Ministério da Saúde, o esforço coletivo, desde o profissional local da unidade de saúde, até a esfera federal no Ministério da Saúde, é imprescindível para que a avaliação possa se tornar orgânica aos processos de trabalho do SUS a ponto de influenciar o seu comportamento ${ }^{19}$.

Além da recusa em participar da pesquisa, não encontrar alguns profissionais e gestores nas unidades de saúde e o pouco tempo de atuação (menos de seis meses) explicam os baixos percentuais de participação. Estes podem ser indicadores do descompasso entre a política oficial do SUS e a gestão do trabalho das equipes de saúde da família, pois a consolidação de processos de gestão demanda tempo de maturação, o que é desfavorecido pela rotatividade de gestores e profissionais de saúde e, certamente, pelo pouco tempo em que estão disponíveis nas unidades de saúde.

\section{Pontos Fortes}

Destacam-se como pontos fortes da pesquisa a seleção aleatória da amostra de usuários da ESF e a comparação das avaliações dos gestores, profissionais e usuários. O confronto das três perspectivas é pouco comum na literatura e possibilitou uma análise ampliada das estruturas e processos que permeiam a consolidação da atenção primária como porta de entrada do SUS.

\section{Resultados}

Dos gestores entrevistados, $62,5 \%$ eram diretores das unidades de saúde, 31,3\% coordenadores de área da ESF e 6,2\% coordenadores centrais da SEMUS. Em relação ao tempo de experiência na função, 56,2\% trabalhavam de 6 a 12 meses. Dos profissionais, $72,5 \%$ eram enfermeiros e $27,5 \%$ médicos; 47,8\% tinham experiência de 13 a 24 meses na ESF. Predominaram usuários do sexo feminino $(72,6 \%)$, faixa etária de 17 a 27 anos $(22,7 \%)$ e com ensino fundamental incompleto (32\%).

O Índice Composto de Utilização dos Serviços verificado foi de 3,96 para gestores, 3,52 para profissionais e 3,66 para usuários, situando a obtenção desse atributo na ESF de São Luís em torno de $60 \%$ (grau insatisfatório). Não houve diferença estatisticamente significativa entre os índices comparando-se os grupos pesquisados (p-valor 0,13) - Tabela 1.

Utilizar serviços preventivos na unidade foi o indicador da dimensão Utilização dos Serviços que obteve maior escore na avaliação dos usuários, exatamente escore 4 , o que significa obtenção desse elemento em $61 \%$ a $80 \%$ da sua totalidade (grau satisfatório). O indicador de menor escore verificado foi utilizar serviços curativos (escore entre 3 e 4 - obtenção de cerca de $60 \%$ grau insatisfatório) - Tabela 1.

Os escores médios atribuídos à realização de consulta na ESF antes de consultar com especialista variaram entre 3 e 4, o que indica obtenção em torno de $60 \%$ dessa característica (grau insatisfatório), Tabela 1.

O Índice Composto de Acesso na avaliação dos gestores foi 2,31, para os profissionais 2,05 e para os usuários 1,72 , verificando-se diferença estatisticamente significante entre as respostas, $\mathrm{p}$-valor $<0,001$. Ao pós-teste de Dunn observou-se que a diferença foi significativa $(\mathrm{p}<0,05)$ entre as avaliações de usuários e gestores e entre profissionais e gestores (Tabela 1).

Para os gestores, profissionais e usuários, não precisar pagar para consultar na unidade de saúde foi o que mais facilitou o acesso, sendo obtido escore médio entre 4 e 5 (obtenção satisfatória próximo de $100 \%$ ).

A disponibilidade de equipamentos adequados na unidade de saúde foi apontada pelos usuários como o segundo indicador melhor avaliado, com escore entre 3 e 4,obtenção de $41 \%$ a $60 \%$, porém em grau insatisfatório (Tabela 1 ).

A possibilidade de conseguir consulta em menos de 24 horas foi apontada pelos gestores e 
Tabela 1. Escores dos indicadores e Índices Compostos de Acesso e Utilização dos Serviços e Índice Total do Primeiro Contato, segundo usuários, profissionais e gestores. São Luís, 2010.

\begin{tabular}{|c|c|c|c|c|c|}
\hline $\begin{array}{c}\text { Dimensão do } \\
\text { primeiro } \\
\text { contato }\end{array}$ & Indicadores & $\begin{array}{c}\text { Escore } \\
\text { usuários }^{*} \\
(\text { IC 95\%) }\end{array}$ & $\begin{array}{c}\text { Escore } \\
\text { profissionais* } \\
(\text { IC } 95 \%)\end{array}$ & $\begin{array}{c}\text { Escore } \\
\text { gestores }^{*} \\
\text { (IC 95\%) }\end{array}$ & $\begin{array}{c}\text { P-valor } \\
\text { Kruskal- } \\
\text { Wallis }\end{array}$ \\
\hline \multirow{4}{*}{$\begin{array}{c}\text { Utilização } \\
\text { dos Serviços }\end{array}$} & Usual para serviços preventivos & $4,00(3,9-4,1)$ & - & - & - \\
\hline & Usual para serviços curativos & $3,33(3,2-3,4)$ & - & - & - \\
\hline & $\begin{array}{l}\text { Porta de entrada para consultas } \\
\text { especializadas }\end{array}$ & $3,64(3,5-3,7)$ & $3,52(3,21-3,82)$ & $3,96(3,47-4,44)$ & 0,47 \\
\hline & $\begin{array}{l}\text { Índice Composto da Utilização } \\
\text { dos Serviços }\end{array}$ & $3,66(3,63-3,68)$ & $3,52(3,21-3,82)$ & $3,96(3,47-4,44)$ & 0,13 \\
\hline \multirow[t]{13}{*}{ Acesso } & $\begin{array}{l}\text { Não tem que deixar de trabalhar parar } \\
\text { consultar }\end{array}$ & $2,38(2,24-2,51)$ & - & - & - \\
\hline & Facilidade em marcar consulta & $3,34(3,2-3,4)$ & - & - & - \\
\hline & $\begin{array}{l}\text { Consegue uma consulta emmenos } \\
\text { de } 24 \text { horas }\end{array}$ & $2,42(2,3-2,5)$ & $3,05(2,76-3,33)$ & $3,65(3,31-3,99)$ & $<0,001$ \\
\hline & $\begin{array}{l}\text { Telefone para marcar consultas } \\
\text { quando a Unidade está aberta }\end{array}$ & $0,72(0,62-0,82)$ & $1,97(1,49-2,44)$ & $2,96(2,23-3,69)$ & $<0,001$ \\
\hline & $\begin{array}{l}\text { Telefone para marcar consultas } \\
\text { quando a Unidade está fechada }\end{array}$ & $0,19(0,11-0,23)$ & $0,45(0,16-0,74)$ & $0,59(0,05-1,13)$ & 0,002 \\
\hline & $\begin{array}{l}\text { Espera por menos de } 30 \text { minutos } \\
\text { antes de ser atendido* }\end{array}$ & $1,05(0,95-1,15)$ & $2,46(2,19-2,74)$ & $2,12(1,67-2,57)$ & $<0,001$ \\
\hline & Medicamentos adequados & $2,36(2,20-2,40)$ & $2,58(2,33-2,82)$ & $2,81(2,40-3,21)$ & 0,061 \\
\hline & Equipamentos adequados & $3,55(3,4-3,6)$ & $2,52(2,28-2,76)$ & $2,75(2,34-3,15)$ & $<0,001$ \\
\hline & $\begin{array}{l}\text { Unidade aberta uma vez por } \\
\text { semana após as } 18 \text { horas }\end{array}$ & $0,16(0,12-0,20)$ & $0,13(0,03-0,22)$ & $0,53(0,13-0,93)$ & 0,002 \\
\hline & Unidade aberta fim de semana & $0,11(0,10-0,21)$ & $0,25(0,12-0,38)$ & $0,37(0,12-0,62)$ & $<0,001$ \\
\hline & $\begin{array}{l}\text { Não precisar de pagamento para } \\
\text { consultar nesta Unidade* }\end{array}$ & $4,85(4,80-4,89)$ & $4,96(4,90-5,00)$ & 5 & 0,124 \\
\hline & Índice Composto do Acesso & $1,72(1,70-1,73)$ & $2,05(1,70-2,39)$ & $2,31(1,74-2,87)$ & $<0,001$ \\
\hline & Índice Total do Primeiro Contato & $2,29(2,18-2,39)$ & $2,19(1,75-2,44)$ & $2,47(1,90-3,03)$ & $<\mathbf{0 , 0 0 1}$ \\
\hline
\end{tabular}

profissionais como a segunda característica da ESF que mais facilita o acesso, sendo a obtenção de $41 \%$ a $60 \%$, grau insatisfatório (Tabela 1 ).

Dispor da unidade de saúde aberta no fim de semana e pelo menos uma vez por semana após as 18 horas foram os dois indicadores pior avaliados pelos três sujeitos da pesquisa. Ambos os indicadores apresentaram escores abaixo de 1 , portanto, percentual de obtenção menor que $0 \%$ a $20 \%$, grau crítico de obtenção (Tabela 1).

Dos indicadores de acesso, apenas não precisar de pagamento para consultar na unidade situou-se em um grau de obtenção satisfatório, os demais ou foram classificados em nível crítico ou insatisfatório para gestores, profissionais e usuários (Tabela 1).

A avaliação dos gestores e profissionais foi significativamente superior $(\mathrm{p}<0,05)$ à dos usuá- rios para sete indicadores de acesso (conseguir consulta em menos de 24 horas, disponibilidade de telefone para marcar consulta quando a unidade está aberta, disponibilidade de telefone para marcar consulta quando a unidade está fechada, espera por menos de 30 minutos antes de ser atendido, disponibilidade adequada de equipamentos, unidade aberta no final de semana, unidade aberta após as 18 horas - Tabela 1), dos nove que eram comuns aos entrevistados. Os indicadores facilidade em marcar consultas e não ter que deixar de trabalhar para consultar foram investigados somente nos usuários. A Figura 1 ilustra as convergências e divergências das respostas dos gestores, profissionais e usuários acerca dos indicadores estudados.

Os usuários avaliaram o atributo acesso também acerca da necessidade de perder trabalho 


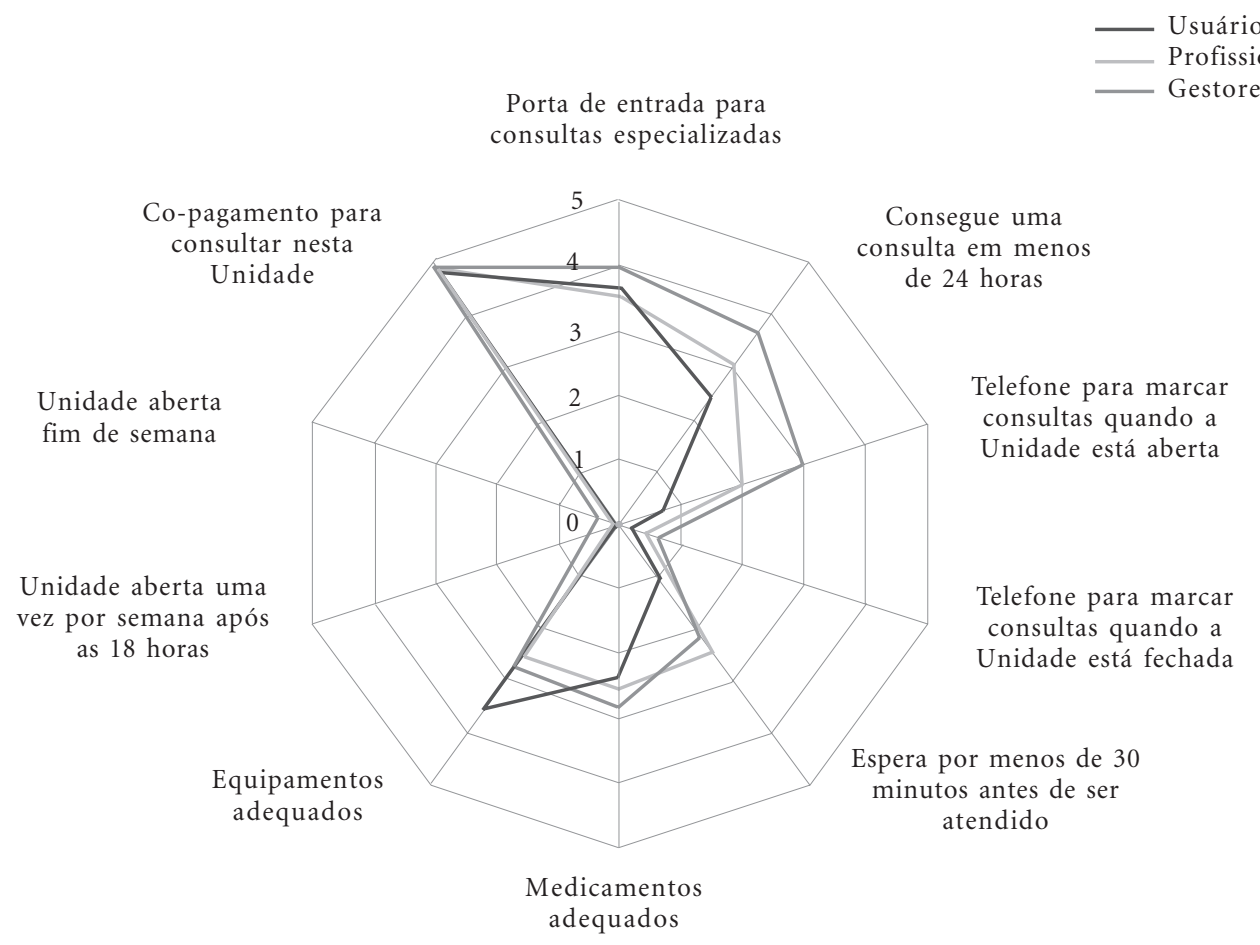

Figura 1. Comparação da avaliação de gestores, profissionais e usuários acerca dos indicadores comuns aos entrevistados nas dimensões acesso e utilização dos serviços na Estratégia Saúde da Família em São Luís, 2010.

para realizar consulta $(3,38)$ e sobre a facilidade de marcar consulta $(4,34)$, apresentando obtenção insatisfatória, conforme Tabela 1 .

A experiência total da utilização dos serviços como porta de entrada do SUS é refletida pelo Índice Total de Porta de Entrada, que se situou entre 3 e 4 na avaliação dos três tipos de entrevistados (obtenção de $41 \%$ a $60 \%$ - insatisfatória). O pós-teste de Dunn demonstrou que foi significativa a diferença entre as respostas dos usuários e gestores e entre as destes e as dos profissionais, sendo as dos gestores as mais favoráveis (Tabela 1).

O alfa de Cronbach, calculado para a utilização dos serviços, foi 0,57 e nenhuma pergunta aumentou o coeficiente com sua retirada da análise. Para o acesso, o alfa de Cronbach observado foi 0,50 para gestores, 0,47 para profissionais e 0,58 para usuários, todos abaixo do valor considerado consistente.

\section{Discussão}

A utilização dos serviços foi mais bem avaliada do que o acesso, o que concorda com os achados de outros estudos ${ }^{9,10}$. A disponibilidade das unidades de saúde da família abertas pelo menos uma vez por semana após as 18 horas e no final de semana foram os aspectos que tiveram pior avaliação. Também demandam melhoria os indicadores relacionados ao tempo de espera pela consulta e disponibilidade de medicamentos, que se mostraram insatisfatórios. Por outro lado, a ESF está sendo utilizada satisfatoriamente para a obtenção de serviços preventivos.

Diferenças nas expectativas de usuários, profissionais e gestores quanto à atenção desenvolvida têm sido verificadas na literatura ${ }^{9,10,20}$, e não foi diferente aqui. Gestores e profissionais avaliaram melhor o atendimento, enquanto os usuários avaliaram melhor a disponibilidade de equipamentos adequados. Essa divergência denota a necessidade de maior aproximação entre gestão, 
serviços de saúde e usuários, assim como o fortalecimento de ações de monitoramento e avaliação local para que as fragilidades e as novas possibilidades sejam identificadas no processo de produção dos serviços de saúde nas equipes de saúde da família.

\section{Avaliação do acesso aos serviços}

A avaliação do acesso mostrou a insuficiência dessa dimensão na ESF em São Luís, o que concorda com estudo realizado por Almeida e Macinko ${ }^{9}$, em Petrópolis, no qual se verificou obtenção de cerca de $20 \%$ da dimensão em questão. Em estudo realizado no estado de São Paulo a acessibilidade também foi um dos atributos que teve menor percentual de obtenção, em torno de $40 \%{ }^{10}$.

\section{Convergências entre gestores, profissionais e usuários}

A Figura 1 demonstra a comparação da avaliação entre gestores, profissionais e usuário, considerando os indicadores comuns aos entrevistados.

Houve convergência entre os três tipos de entrevistados na avaliação da disponibilidade de medicamentos adequados e não precisar de pagamento para obtenção de serviços (Figura $1 \mathrm{e}$ Tabela 1).

Acerca da disponibilidade dos medicamentos adequados, essa é uma característica alcançada insatisfatoriamente na ESF de São Luís. O mesmo percentual de obtenção dessa característica foi verificado em Petrópolis, em 2006, onde também foi observada semelhança entre as respostas de gestores, profissionais e usuários, com percentuais de obtenção de cerca de $40 \%{ }^{9}$.

Os medicamentos distribuídos na APS são os essenciais, que, segundo a Organização Mundial da Saúde, são aqueles que satisfazem as necessidades de cuidados de saúde básica da maioria da população e devem estar acessíveis em dose certa a toda a sociedade ${ }^{21}$. Portanto, a falta de acesso aos mesmos implica fragilização do processo de cura, reabilitação e prevenção de doenças. É possível que a falta de planejamento das compras e de eficiente sistema de controle e informação, aliado à demora no processo de aquisição, possa estar contribuindo para o aumento de gastos com aquisição de medicamentos, reduzindo a qualidade dos serviços ${ }^{22}$.

Não precisar pagar para obter consulta foi o indicador de acesso melhor avaliado pelos três tipos de entrevistados, o que era esperado, considerando que a gratuidade é característica basilar do SUS.

\section{Divergências entre gestores e profissionais com relação aos usuários}

A consecução de consulta em menos de 24 horas, espera por menos de 30 minutos, disponibilidade de equipamentos adequados e de telefone para marcar consulta quando a unidade está aberta ou fechada foram avaliados de forma diferente pelos entrevistados. Gestores e profissionais concordaram em todos os indicadores e divergiram dos usuários. Os usuários fizeram pior avaliação de todos os indicadores, exceto da disponibilidade de equipamentos (Gráfico 1). Os usuários avaliaram que a facilidade em marcar consulta é insatisfatória e sua expectativa sobre a possibilidade de conseguir consulta em 24 horas, além de insatisfatória, é menor que a dos profissionais e gestores. Este resultado concorda com as verificações de Almeida e Macinko ${ }^{9}$, que encontraram percentuais de obtenção de $21 \%$ a $40 \%$ para facilidade em marcar consulta e de $41 \%$ a $60 \%$ para possibilidade de marcação de consulta em 24 horas. Em pesquisa qualitativa sobre acesso, Azevedo e Oliveira ${ }^{14,23}$ observaram que o principal determinante da não utilização dos serviços da ESF foi a dificuldade em marcar consultas.

É importante verificar como está se dando o processo de marcação de consultas, assim como o tempo de consecução do atendimento. Nessa verificação se faz importante considerar a possibilidade de os usuários atribuírem valoração negativa ao poder dos agentes comunitários de saúde em marcar consultas, encarando-se esse fato como uma forma geradora de privilégios para as pessoas mais próximas destes ${ }^{24}$. Impõe-se ainda como indicador que necessita de melhoria o tempo de espera por atendimento.

Provavelmente a falta de domínio sobre os conhecimentos técnicos da assistência fez com que usuários avaliassem melhor que os profissionais e gestores a disponibilidade de equipamentos adequados nas unidades, uma vez que os profissionais, que têm necessidade direta dos equipamentos no seu processo de trabalho, e os gestores, que supervisionam o processo de trabalho e o provimento de equipamentos às unidades, avaliaram esse indicador em um grau significativamente menos satisfatório.

O funcionamento das USF no final de semana e após as 18 horas foram os indicadores que apresentaram pior avaliação pelos entrevistados. 
Os usuários também avaliaram que, muitas vezes, a perda do dia de trabalho é necessária para obtenção de consulta. O planejamento acerca dos horários de funcionamento precisa ser revisto a fim de possibilitar aos usuários o acesso aos serviços em horário alternativo ao seu horário de trabalho (final de semana ou após as 18 horas). A agenda aos sábados é um exemplo do esforço no sentido de possibilitar acessibilidade e maior utilização da APS como primeiro contato com o sistema de saúde ${ }^{1}$. A ESF, ao pretender reorientar a atenção à saúde no SUS, precisa ter suas unidades funcionando de acordo com a necessidade do usuário, em parceria com as comunidades, para diminuir as barreiras de acesso e melhorar a utilização dos serviços.

Nessa perspectiva, a participação social em saúde não pode estar restrita às instâncias formalizadas como Conselhos e Conferências de Saúde. Esta deve ser valorizada e incentivada no dia-a-dia dos serviços do SUS, para que se constitua em um espaço no qual os usuários dos serviços possam, além de verbalizar suas necessidades, influenciar os processos de trabalho nas equipes de saúde da família ${ }^{24}$.

\section{Avaliação da utilização dos serviços}

A utilização da ESF para obtenção de serviços preventivos foi avaliada em grau satisfatório, em conformidade com a Política Nacional de Atenção Básica ${ }^{25}$, que aponta que a ESF deve ser centrada na promoção e proteção da saúde. Não houve divergências na avaliação dos gestores, profissionais e usuários. Deve-se levar em consideração que poucos indicadores foram considerados e apenas um deles (necessidade de consulta na unidade básica antes de consultar especialista) foi avaliado simultaneamente pelos gestores, profissionais e usuários.

As mulheres utilizaram os serviços de saúde em maior percentual que os homens. Este fato infere uma situação social em que a mulher tem papel nuclear no grupo familiar no cuidado à saúde, conforme Ibãnez et al. ${ }^{10}$, e confirma que as mulheres utilizam mais os serviços de saúde do que os homens ${ }^{8,26}$. Isto reforça a necessidade de investir na qualificação da atenção à saúde masculina no âmbito da ESF em São Luís. A desigualdade de gênero no uso dos serviços indica tanto diferenças no comportamento dos indivíduos, quanto características da oferta de serviços que cada sociedade disponibiliza para seus membros ${ }^{27}$. Os homens buscam menos a atenção primária e, muitas vezes, acabam utilizando a atenção especializada para questões sensíveis à atenção primária, invertendo a lógica da ESF como porta de entrada preferencial para o SUS ${ }^{26}$. Além da fragilização da atenção primária como porta de entrada para o SUS, esta situação pode acarretar sobrecarga dos serviços de urgência com casos não urgentes como resultado da busca por atenção à saúde do homem que trabalha durante o dia e, portanto, não consegue acessar os serviços disponíveis nesse horário.

Há uma década foi apontado em estudo da implementação da ESF em dez grandes centros urbanos que, nas grandes cidades, é necessário estudar como alternativa a atuação das equipes em locais próximos ao local de trabalho da população adscrita ou estender o horário de atendimento, pois a adscrição domiciliar dificulta a provisão de assistência para trabalhadores que, em geral, tem seu lugar de trabalho distante do local de moradia ${ }^{28}$. Entretanto, até o momento não se verificam grandes avanços nessa perspectiva em nível nacional.

\section{Considerações finais}

O atributo porta de entrada foi avaliado como insatisfatório na ESF, portanto com grande necessidade de melhora. Entretanto, apesar de considerada insatisfatória, as pessoas utilizam os serviços da ESF para entrar no SUS. Este achado pode apontar para a necessidade de mais investigações para saber se existe por parte da população adscrita o real reconhecimento das Unidades de Saúde da Família como fonte regular de atenção, ou se a utilização da Unidade se dá simplesmente pela restrição de opções, situação esta também não esclarecida nos estudos citados neste trabalho.

A implantação da ESF em São Luís é parcial e seu devido funcionamento como ponto de acesso preferencial ao SUS precisa de consideráveis melhorias nas estruturas e processos descritos neste trabalho. Possibilitar a abertura das unidades nos finais de semana e após as 18 horas em alguns dias da semana pode ser uma alternativa para que os usuários possam acessar melhor os serviços. Como as unidades funcionam em horário comercial o acesso é favorecido às crianças e mulheres fora da população economicamente ativa e dificultado para quem está no mercado de trabalho, principalmente para quem trabalha de forma autônoma ou no setor informal.

Pesquisa realizada pelo IBGE em 2003 apontou que mais da metade das microempresas bra- 
sileiras fazem parte do mercado informal, e grande parte das empresas pertencia a trabalhadores por conta própria, sendo que os homens correspondiam a $64 \%$ das pessoas ocupadas nelas ${ }^{29}$. Em São Luís, 65,8\% das unidades domiciliares tem o homem como único responsável ${ }^{30}$.

Portanto, além da questão de gênero, a não disponibilidade de unidades de saúde da família abertas após o horário de trabalho ou finais de semana pode estar contribuindo para a inversão da lógica, uma vez que se constituem como barreira de acesso aos serviços para o trabalhador.
Identificar os elementos que tem dificultado a disponibilidade adequada de medicamentos, diminuir o tempo de espera pelas consultas e estruturar melhor as unidades de saúde certamente são estratégias que tornariam a acessibilidade favorável à utilização da estratégia saúde da família como porta de entrada do SUS em São Luís. Para tanto, se faz necessário aproximar gestores, profissionais e usuários a fim de dirimir as divergências de concepções e expectativas. Uma alternativa é fortalecer os espaços de participação social nas unidades de saúde da família.

\section{Colaboradores}

RS Reis, LC Coimbra, AAM Silva, AM Santos, MTSSB Alves, ZC Lamy, SVO Ribeiro, MSA Dias e RA Silva participaram igualmente de todas as etapas de elaboração deste artigo.

\section{Agradecimentos}

Agrademos à Fundação de Amparo à Pesquisa e ao Desenvolvimento Científico e Tecnológico do Maranhão pelo financiamento desta pesquisa e ao Programa de Pós-Graduação em Saúde Coletiva da Universidade Federal do Maranhão por proporcionar aos seus alunos de mestrado e doutorado a participação nas Oficinas de Redação de Artigos Científicos, que enriqueceram esta produção. 


\section{Referências}

1. Brasil. Ministério da Saúde (MS). Conselho Nacional de Secretários Estaduais de Saúde (CONASS). Atenção Primária e Promoção da Saúde. Brasília: CONASS; 2007.

2. Starfield B. Atenção primária: equilíbrio entre necessidades de saúde, serviços e tecnologia. Brasília: Organização das Nações Unidas para a Educação, a Ciência e a Cultura, Ministério da Saúde; 2002.

3. Giovanella L, Ecorel S, de Mendonça MHM. Porta de entrada pela atenção básica? Integração do PSF à rede básica de saúde. Saúde em Debate 2003; 27(65): 278-289.

4. Brasil. Ministério da Saúde (MS). Documento Final da Comissão de Avaliação de Atenção Básica. Brasília: MS; 2003.

5. Aguiar RAT. A construção internacional do conceito de Atenção Primária à Saúde (APS) e sua influência na emergência e consolidação do Sistema Único de Saúde no Brasil, 2003 [dissertação]. Belo Horizonte: Universidade Federal de Minas Gerais; 2003.

6. Harzheim E, Stein AT, Álvarez-Dardet C. A efetividade dos atributos da atenção primária sobre a saúde infantil. Cien Saude Colet 2004; 18(1):23-40.

7. Donabedian A. The quality of medical care. Science 1978; 200(4344):856-864.

8. Travassos C, Martins M. Uma revisão sobre os conceitos de acesso e utilização de serviços de saúde. Cad Saude Publica 2004; 20(Supl. 2):S190-198.

9. Almeida C, Macinko J. Validação de uma metodologia de avaliação rápida das características organizacionais e do desempenho dos serviços de atenção básica do Sistema Único de Saúde (SUS) em nível local. In: Almeida C, Macinko J. Mecanismos institucionais de monitoramento e avaliação da atenção básica. Brasília: Organização Pan-Americana de Saúde (OPAS/OMS), Ministério da Saúde; 2006. (Série Técnica Desenvolvimento de Sistemas e Serviços de Saúde)

10. Ibãnez N, Rocha JSY, Castro PC, Riobeiro MCSA, Forster AC, Novaes MHD, Viana AL. Avaliação do desempenho da Atenção Básica no Estado de São Paulo. Cien Saude Colet 2006; 11(3):683-703.

11. Brasil. Ministério da Saúde (MS). Cadastro Nacional de Estabelecimentos de Saúde. Número de equipes de Saúde da Família. [página na Internet]. 2010. [acessado 2010 jan 22]. Disponível em: http://cnes. datasus.gov.br/.

12. Instituto Brasileiro de Geografia e Estatística (IBGE). Censo. [página na Internet]. 2010. [acessado 2010 jan 22]. Disponível em http://www.ibge.gov.br/.

13. Harzhein E, Starfield B, Rajmil L, Alvarez-Daret C, Stein AT. Consistência interna e confiabilidade da versão em português do Instrumento de avaliação da atenção primária (PCATool-Brasil) para serviços de saúde infantil. Cad Saude Publica 2006; 22(8):1649-1659.

14. Oliveira MMC. Presença e extensão dos atributos da atenção primária em Porto Alegre: uma análise agregada [dissertação]. Porto Alegre: Universidade Federal do Rio Grande do Sul; 2007.

15. Shi L, Starfield B, Xu J. Validating the Adult Primary Care Assessment Tool. The Journal of Family Practice 2001; 50(2):161-175.
16. Hair JE, Anderson RE, Tatham RL, Black WC. Análise multivariada de Dados. Porto Alegre: Bookman; 2005.

17. Pasquali L. Psicometria: Teoria dos testes na psicologia e educação. Petrópolis: Vozes; 2003.

18. Brasil. Ministério da Saúde (MS). Conselho Nacional de Saúde. Resolução nº 196 de 10 de outubro de 1996. Diretrizes e Normas Regulamentadoras de Pesquisas Envolvendo Seres Humanos. Diário Oficial da União 1996; 16 out.

19. Brasil. Ministério da Saúde (MS). HUMANIZA SUS. Gestão Participativa. Co-gestão. Brasília: MS; 2004.

20. Novaes HMD. Pesquisa em, sobre e para os serviços de saúde: panorama internacional e questões para a pesquisa em saúde no Brasil. Cad Saude Publica 2004; 20(Supl. 2):147-173.

21. Organización Mundial de la Salud (OMS). Selección de medicamentos esenciales. Perspectivas Políticas sobre Medicamentos de la OMS. Ginebra: OMS; 2002.

22. Brasil. Ministério da Saúde (MS). Departamento de Assistência Farmacêutica e Insumos Estratégicos. Aquisição de medicamentos para a assistência farmacêutica no SUS. Orientações Básicas. Brasília: MS; 2006.

23. Azevedo ALM. Acesso à atenção à saúde no SUS: o PSF como (estreita) porta de entrada [dissertação]. Recife: Fundação Oswaldo Cruz; 2007.

24. Nunes MO, Trad LB, Almeida BA, Homem CR, Melo MCIC. O agente comunitário de saúde:construção da identidade desse personagem híbrido e polifônico. Cad Saude Publica 2002; 8(6):1639-1646.

25. Brasil. Ministério da Saúde (MS). Portaria nº 648/ GM, de 28 de março de 2006. Diário Oficial da União 2006; 29 mar.

26. Brasil. Ministério da Saúde (MS). Política Nacional de Atenção Integral à Saúde do Homem. 2008. [página na Internet]. [acessado 2011 mar 12]. Disponível em: http://dtr2001.saude.gov.br/sas/PORTARIAS/ Port2008/PT-09-CONS.pdf

27. Travassos C. Utilização dos serviços de saúde no Brasil: gênero, características familiares e condição social. Rev Panam Salud Publica 2002; 11(5):365-373.

28. Escorel S, coordenador. Avaliação da implementação do Programa Saúde da Família em dez grandes centros urbanos: síntese dos principais resultados. Brasília: Ministério da Saúde (MS), Departamento de Atenção Básica; 2002.

29. Instituto Brasileiro de Geografia e Estatística (IBGE). Economia Informal Urbana 2003. [página na Internet]. [acessado 2011 abr 15]. Disponível em: http:/ /www.ibge.gov.br/home/estatistica/populacao/ mapa_mercado_trabalho/default.shtm.

30. Instituto Brasileiro de Geografia e Estatística (IBGE). Censo Demográfico 2012: Características da População e dos Domicílios: Resultados do Universo. [página na Internet]. [acessado 2011 abr 15]. Disponível em: http://www.ibge.gov.br/cidadesat/topwindow. htm?1

Artigo apresentado em 31/07/2012

Aprovado em 30/11/2012

Versão final apresentada em 21/12/2012 
